\title{
ANÁLISIS
}

\section{STAKEHOLDERS O CÓMO APLICAR LA TEORÍA DE LOS GRUPOS DE INTERÉS EN LAS BIBLIOTECAS PÚBLICAS}

\section{Ana Ríos-Hilario y João De-Sousa-Guerreiro}
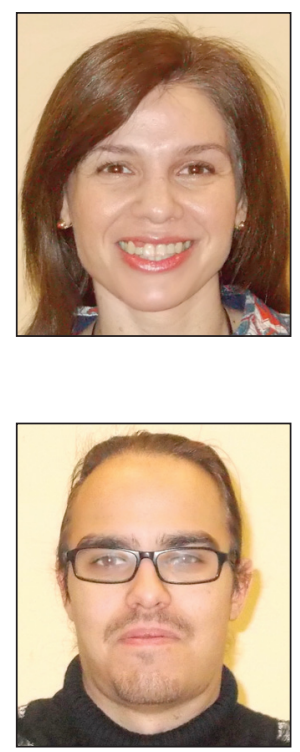

\section{Resumen}

Ana Ríos-Hilario es doctora en documentación y profesora del Departamento de Biblioteconomía y Documentación de la Universidad de Salamanca. Su actividad investigadora se ha centrado tanto en la descripción documental como en el estudio y aplicación de las metodologías y técnicas de investigación en el campo de la información y la documentación, dirigiendo y participando en varios proyectos de investigación de muy diverso ámbito y siendo autora de varias publicaciones sobre ese mismo tema. Es asesora del proyecto de promoción de la participación ciudadana que se está desarrollando en la Biblioteca Pública de Salamanca.

http://orcid.org/0000-0002-7052-5860

anarihi@usal.es

João De-Sousa-Guerreiro es licenciado en ciencias de la información por la Universidad de Coimbra y alumno del doctorado en información y documentación en la Universidad de Salamanca. Trabajó como gestor cultural en diversas bibliotecas y centros culturales. Actualmente es coordinador de un proyecto de promoción de la participación ciudadana como elemento básico de la gestión de servicios culturales en la Biblioteca Pública de Salamanca, donde se ensaya un modelo de relación con los principales actores sociales de la comunidad.

http://orcid.org/0000-0002-9417-0996

joaoguerreiro@usal.es

Universidad de Salamanca, Departamento de Biblioteconomía y Documentación Francisco de Vitoria, 6-16. 37008 Salamanca, España

La teoría de los stakeholders está directamente relacionada con disciplinas como el marketing y movimientos como la participación ciudadana, ambos de vital importancia para la situación que atraviesan las bibliotecas públicas del siglo XXI. Nuestro propósito ha sido definir un modelo de relación con los principales grupos de interés pertenecientes a las citadas instituciones. Una vez establecido el marco teórico, se define el objeto de estudio -los stakeholders o grupos de interés- para posteriormente justificar su uso en el entorno bibliotecario. A continuación se detallan las tres fases de las que consta el modelo propiamente dicho: identificación, priorización y relación con los stakeholders. Por último, se presenta una serie de ejemplos prácticos. Como conclusión se expone que es necesario que estos movimientos se basen en la investigación científica, y adapten y apliquen las técnicas y principios que permitan realmente la relación entre los stakeholders y la institución.

\section{Palabras clave}

Teoría de los stakeholders; Participación ciudadana; Grupos de discusión; Bibliotecas públicas; Modelo de relación; Marketing.

\section{Title: Stakeholders how to apply the theory of interest groups in public libraries}

\begin{abstract}
Stakeholder theory is directly related to disciplines such as marketing and to trends such as citizen participation, both vital to the current situation of the public libraries of the XXI century. Our aim has been to define a relationship model showing the leading stakeholders for these institutions. Having established the theoretical framework, the stakeholder concept is defined and its applicability to the library environment is described. Afterwards the three phases that constitute the model are detailed: identification, prioritization and relationship with stakeholders. Finally, a series of practical examples is presented. The conclusion is that stakeholder models must be based on scientific research, and must adapt and apply interest group techniques and principles that truly build relationships between stakeholders and the library.
\end{abstract}

\section{Keywords}

Stakeholders theory; Citizens participation; Focus groups; Public library; Relationship model; Marketing. 
Ríos-Hilario, Ana; De-Sousa-Guerreiro, João (2015). “Stakeholders o cómo aplicar la teoría de los grupos de interés en las bibliotecas públicas”. El profesional de la información, v. 24, n. 1, enero-febrero, pp. 71-76.

http://dx.doi.org/10.3145/epi.2015.ene.09

\section{Introducción}

Desde su aparición como disciplina científica a principios del siglo XX, el concepto de marketing ha evolucionado y cambiado considerablemente de perspectiva según el contexto social, económico y tecnológico. Por tal motivo, su objetivo se ha ido modificando: originalmente su función fue distribuir el producto al mayor número de personas; posteriormente se pretendía estimular la compra; y en la actualidad todo se orienta a identificar y satisfacer las necesidades y expectativas del mercado.

Se puede definir el marketing como "la función de la empresa responsable de conocer, conquistar y mantener clientes, gracias a una satisfacción constante de sus necesidades y a una creación de relaciones positivas e innovadoras" (BelioGalindo; Sanz-Andrés, 2007). En un primer momento la relación entre empresa y consumidores consistía en recoger información sobre las expectativas y necesidades de los mismos. Posteriormente se tuvo en cuenta la importancia de una verdadera relación bilateral, así los clientes no solamente exponen sus necesidades, sino que participan en la creación de los productos y servicios de la empresa mediante estrategias participativas.

\section{La Teoría de los stakeholders (Freeman,} 1984), propone que se tenga en cuenta la opinión de los actores sociales con los que se relaciona la institución-stakeholders o grupos de interés- y que las decisiones tomadas sean ventajosas para todos

El actual contexto económico ha provocado cierta inseguridad social ocasionando cambios en la gestión de las empresas. La aportación más significativa fue la Teoría de los stakeholders (Freeman, 1984), que de una forma sucinta propone que se tenga en cuenta la opinión de los actores sociales con los que se relaciona la institución-stakeholders o grupos de interés-y que las decisiones tomadas sean ventajosas para todos. Dicha teoría está directamente relacionada con la responsabilidad social corporativa que promueve que las organizaciones sean responsables por sus acciones y que ponderen su impacto en el tejido social. Actualmente también las instituciones públicas, procuran ser responsables socialmente, es decir, que sus actividades sean a favor de la comunidad y de modo progresivo más transparentes y sostenibles según el nuevo contexto económico-social.

Las bibliotecas públicas siempre han estado relacionadas con las comunidades a las que sirven, y actualmente sufren el impacto de la crisis económica. Además, existe la creencia de que las bibliotecas públicas ya no son necesarias porque los usuarios pueden satisfacer sus necesidades de información en internet. Estos hechos han afectado gravemente a dichas instituciones tanto en su desempeño laboral como en la imagen que los ciudadanos perciben de ellas. Esta situación ha propiciado el debate sobre la función de las bibliotecas públicas del siglo XXI. Uno de los caminos que pueden tomarse es el de la participación ciudadana: que sean los grupos de interés conjuntamente con la dirección de la institución quienes construyan la biblioteca que ellos necesitan. Por tal motivo, la biblioteca pública tiene que promocionar la participación ciudadana y crear las relaciones pertinentes con estos grupos teniendo en cuenta los principios y técnicas de la "teoría de los stakeholders".

La participación ciudadana en las bibliotecas públicas se puede entender "como la intervención de la sociedad civil en el ejercicio de las funciones de administración del servicio público y un medio de expresión e integración de las demandas ciudadanas en la consecución de un interés general, un incentivo para una gestión pública eficiente y eficaz" (Conamaj, 2011).

Tomando como base las anteriores premisas, el objetivo del presente artículo es la elaboración de un modelo de relación con los principales grupos de interés de las bibliotecas públicas. Este modelo está basado en las tres fases de la "teoría de los stakeholders" pero, al provenir ésta del ámbito empresarial, se ha realizado una adaptación específica al contexto bibliotecario.

\section{Uno de los caminos que pueden tomar las bibliotecas públicas para redefinir su función es el de la participación ciudada- na: que sean los grupos de interés con- juntamente con la dirección de la insti- tución quienes construyan la biblioteca que necesitan}

Tras una introducción en la que se establece la relación de la "teoría de los stakeholders" con el marketing y la participación ciudadana, el artículo se estructura en los siguientes apartados. En primer lugar, se define el término stakeholders o grupos de interés para, posteriormente, justificar por qué la biblioteca pública debe relacionarse con estos grupos. A continuación se define el modelo propiamente dicho mediante el establecimiento de tres fases: identificación, priorización y relación con los stakeholders. En el último apartado se exponen tres ejemplos representativos de la aplicación de esta teoría que, a su vez, utilizan diferentes técnicas. 


\section{2. ¿Qué son los}

\section{stakeholders?}

Freeman (1984), introdujo el término de stakeholders en el ámbito de la gestión empresarial. Desde un primer momento definió el concepto como "cualquier grupo o individuo identificable que puede afectar o ser afectado por el logro de los objetivos de una organización".

De este modo las organizaciones "no son entidades autónomas con una identidad básica definida independiente y separadamente", sino que es la red de relaciones que mantiene la organización la que la define (Wicks; Gilbert; Freeman, 1994). Aunque teóricamente podamos dibujar un mapa de stakeholders de un sector en general o de una determinada biblioteca en específico (figura 1), no existe un conjunto de grupos de interés pre-definidos de las organizaciones de una determinada área. Por eso, para relacionarse con los stakeholders es necesario aplicar un modelo de relación como el que aquí se propone.

\section{3. ¿Por qué la biblioteca debe relacionarse con su entorno?}

Todas las bibliotecas mantienen algún tipo de relación ya sea formal o informal con sus grupos de interés. La cuestión es mantener una relación planificada con estos grupos para potenciar la misma. Así se pretende que los grupos de interés pasen de una actitud pasiva a una colaboración activa con la biblioteca. La participación es uno de los pilares de la "teoría de los stakeholders", es uno de sus principales objetivos.

Antes de relacionarse con los stakeholders hay que averiguar quiénes son éstos. La biblioteca tiene la oportunidad de reflexionar sobre las personas con las que se relaciona para desarrollar su trabajo diario. Subyace también a la "teoría de los stakeholders" la selección de los grupos de interés más relevantes lo cual permite segmentar de una forma rápida, económica y específica nuestro "mercado", como se detallará en el siguiente apartado.

Además, las relaciones permiten conocer el entorno interno y externo de la biblioteca, a través de sus principales actores. Este hecho facilita comprender un contexto que actualmente es complejo y cambiante y, consecuentemente, ayuda a encontrar oportunidades estratégicas y nuevas formas de resolver los problemas, es decir, crear una biblioteca pública hecha por y para los ciudadanos a partir de conocer sus necesidades.

El contacto directo con ellos permite que conozcan de manera más cercana la biblioteca y las condiciones de trabajo en las que se desenvuelve, lo que propicia una mayor confianza y, por lo tanto, una mejora de la reputación de la biblioteca.

\section{Modelo de relación con los stakeholders de la biblioteca pública}

La "teoría de los stakeholders" se compone de tres fases: "identificar (a través de varios cauces), priorizar (en función de su relevancia) y dar respuesta (estableciendo recursos y medidas necesarias) a las necesidades y demandas de los grupos de interés" (Granda-Revilla; Trujillo-Fernández, 2011). A continuación expondremos de forma individual cada una de las fases explicando las decisiones metodológicas tomadas para adaptar dicha teoría al ámbito bibliotecario.

\subsection{Primera fase: identificación}

El objetivo final de la identificación es crear una lista lo más exhaustiva posible de todas aquellas personas que se relacionan o podrían relacionarse con la biblioteca. En la práctica se lleva a cabo una reunión con las diferentes categorías de los trabajadores de la biblioteca. En primer lugar se explica qué son los stakeholders y las ventajas de relacionarse con ellos. Posteriormente los empleados pasan a identificar los grupos y personas con las que se relaciona la biblioteca. Esta fase suele sorprender a los propios trabajadores que por primera vez ven de forma clara todo el trabajo desarrollado por la institución y la red de relaciones necesarias de la misma. Cuando se tiene la lista de los stakeholders se deben clasificar en categorías:

Tabla 1. Ejemplo de la tabla que resulta de la identificación de stakeholders en la BP de Salamanca

\begin{tabular}{|l|l|}
\hline \multicolumn{2}{|c|}{ Identificación de stakeholders } \\
\hline Categoría & stakeholders \\
\hline \multirow{3}{*}{$\begin{array}{l}\text { Grupos/asociaciones } \\
\text { culturales }\end{array}$} & Pentadrama: asociación de poetas locales \\
\cline { 2 - 2 } & Malhablada: espacio de micro-teatro \\
\cline { 2 - 2 } & Casa de Argentina de Salamanca \\
\hline \multirow{2}{*}{$\begin{array}{l}\text { Grupos/asociaciones } \\
\text { sociales }\end{array}$} & Cruz Roja \\
\cline { 2 - 2 } Proveedores & Asociación de enfermos de leucemia \\
\hline \multirow{2}{*}{} & Librería Nueva Plaza \\
\cline { 2 - 2 } & Librería Cervantes \\
\hline
\end{tabular}




\subsection{Segunda fase: priorización}

La primera fase suele dar como resultado listas bastantes extensas. Debido a que no es posible contactar directamente con un gran número de grupos de interés, se debe realizar una selección de los más relevantes en ese momento según unos criterios que deben estar definidos previamente. Hay que tener en cuenta que la biblioteca no siempre se relacionará con los mismos stakeholders, ya que éstos suelen cambiar con el tiempo según evoluciona el entorno social de la institución. Son varios los criterios que se pueden emplear en el momento de la selección. En el presente modelo se optó por los siguientes:

- Influencia: aquellos que tienen mayor impacto en el logro de los objetivos de la biblioteca.

- Dependencia: aquellos que se ven más afectados positivamente o negativamente por la actividad de la biblioteca.

La biblioteca pública es un servicio abierto a todos los ciudadanos, motivo por el que se ha de tener en cuenta la bilateralidad de las relaciones. Este hecho significa que uno de los criterios debe medir la influencia que tienen los stakeholders en la biblioteca; y, el otro, la influencia que tiene la biblioteca sobre los mismos.

Para llevar a cabo la segunda fase del presente modelo se vuelve a reunir a los trabajadores de la institución. Primeramente, se debaten y registran las características positivas y negativas de cada categoría de stakeholder. Se trata de una pre-evaluación que posteriormente facilitará la priorización propiamente dicha. A continuación con los criterios señalados anteriormente se crea un cuadro que representa gráficamente el lugar que ocupa cada categoría.

Al final de la segunda fase tendremos un conjunto de 3 ó 4 grupos de interés, que serán los más relevantes -alta influencia y alta dependencia- para la biblioteca en ese momento.

Tabla 2. Ejemplo del cuadro que resulta de la priorización de los stakeholders

\begin{tabular}{|c|c|c|c|}
\hline \multicolumn{4}{|c|}{ Priorización de stakeholders } \\
\hline \multirow{4}{*}{ Dependencia } & \multicolumn{3}{|c|}{ Influencia } \\
\hline & \multicolumn{2}{|r|}{ Baja } & Alta \\
\hline & Alta & $\begin{array}{l}{ }^{*} \text { Grupos/ } \\
\text { asociaciones } \\
\text { culturales }\end{array}$ & $\begin{array}{l}\text { *Usuarios } \\
\text { *Empleados }\end{array}$ \\
\hline & Baja & & $\begin{array}{l}\text { *Grupos/Asociaciones } \\
\text { culturales locales }\end{array}$ \\
\hline
\end{tabular}

\subsection{Tercera fase: relaciones}

La tercera fase, y tal vez la más importante, es la relación con los stakeholders. Existen muchos métodos para relacionarse con los grupos de interés, como se puede observar en la obra "El compromiso con los stakeholders" (AccountAbility, 2006). La mayoría de las técnicas se caracterizan por ser cualitativas y grupales. La dinámica grupal simula el ambiente más propicio para la conversación, lo que permite un mayor grado de espontaneidad en el discurso de los participantes, que otras técnicas como pueden ser, por ejemplo, la entrevista individual.

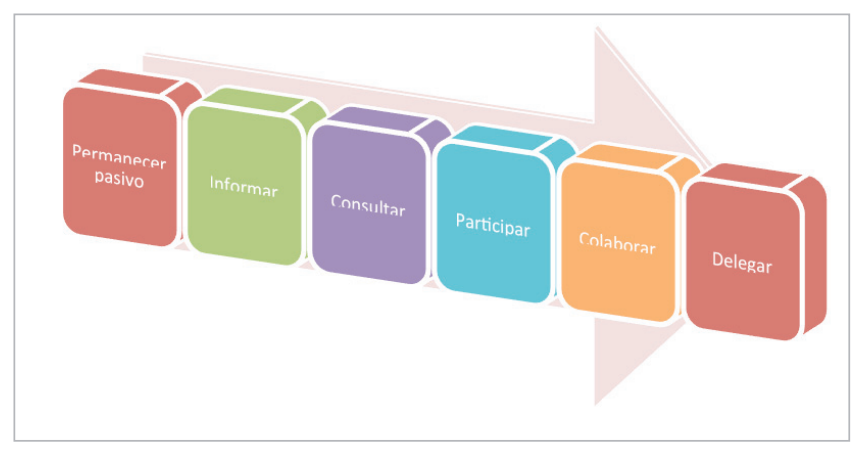

Figura 2. Niveles de relación

La relación con los stakeholders tiene un triple objetivo:

- Recoger información que apoye la toma de decisiones: al obtener la opinión directamente de los grupos de interés, aumenta la probabilidad de que las mejoras o los nuevos servicios ofertados sean aquellos que la comunidad realmente necesita o demanda.

- Crear una dinámica participativa de tal modo que los stakeholders se sientan parte de la institución. El trato directo con los grupos de interés propicia el acercamiento entre ambas partes lo que conlleva un aumento de la reputación de la institución por parte de los grupos de interés.

- Desarrollar el nivel de relación: que los grupos de interés pasen de una posición pasiva a una participativa. Tal y como se observa en la figura 2 se produce una evolución que pasa por varios estados, desde el nivel básico en el que no se propicia ningún tipo de relación hasta el grado máximo de implicación que sería la delegación de algún servicio o actividad a un determinado grupo de interés.

La relación con los grupos de interés está directamente asociada a la planificación estratégica -conocer las expectativas y necesidades de nuestra comunidad y satisfacerlas- y consecuentemente al plan estratégico como herramienta que estructura y guía el trabajo de la organización. En el caso de las bibliotecas que aplican la "teoría de los stakeholders", los planes estratégicos suelen ser el resultado de un conjunto de reuniones y otras actividades de carácter participativo, donde se recoge la opinión de los interesados y se pondera cómo dar respuesta a sus demandas a través del trabajo diario de la biblioteca. Al convocar a los grupos de interés a colaborar en la creación del plan estratégico, éstos ven en esta acción algo más que la "típica" evaluación de los servicios - muchas veces hecha por encuestas- pues su opinión se reflejará en un documento oficial, que sirve en este caso como una muestra de intenciones, un contrato tácito entre éstos y la biblioteca.

Todo este proceso está relacionado con la participación ciudadana, que podemos definir como la inclusión de los grupos que constituyen la sociedad en la toma de decisiones, un tema complejo que debe tratarse escalando los peldaños de la relación de la biblioteca con su comunidad. La toma de decisiones se debe basar en la información: que los grupos conozcan el papel y el trabajo de la biblioteca y que su opinión se centre en una tarea colaborativa y proactiva. Sin una verdadera relación, se corre el riesgo de la crítica fácil y en 
un conjunto de propuestas inviables (por las características de la institución y los recursos de la misma) o en un enfrentamiento entre los deseos opuestos de los diferentes grupos que la biblioteca no podrá satisfacer en su totalidad. Por estos motivos es necesario introducir los grupos de interés en la toma de decisiones de forma progresiva (evaluando la relación, informando sobre la institución, promoviendo la participación, etc.) hasta llegar a delegar algunos servicios y actividades a los mismos. De este modo podrá lograrse el propósito máximo de los movimientos de participación ciudadana y las instituciones abiertas a estos movimientos: una biblioteca hecha por y para la comunidad.

\section{Ejemplos prácticos}

Se han seleccionado tres ejemplos representativos en la aplicación de la teoría de los stakeholders desarrollados en bibliotecas anglosajonas, ya que es en estas zonas donde existe una mayor tradición a la hora de utilizar los métodos participativos además de tener disponible en su portal web la documentación referente a los mismos.

Al seleccionar los ejemplos se intentó presentar tres casos que utilizasen distintas técnicas para relacionarse con los stakeholders y recoger su opinión. Se tuvo en cuenta, también, que los ejemplos representasen tres "estados" diferentes de implicación de los grupos de interés en la toma de decisiones, ordenados de forma creciente. Así la primera biblioteca, Washington Public Library, solamente utiliza la opinión de los stakeholders para determinar los focos de actividad de la biblioteca. El segundo ejemplo, Bellingham Public Library, da un paso más en la relación y recoge información sobre la visión y los valores futuros de la institución, además de tratar con los grupos de interés sobre cuatro dimensiones esenciales a tener en cuenta en la planificación estratégica. Finalmente, la Toronto Public Library es un caso ejemplar donde existen varios grados de relación: desde el análisis de los comentarios en los blogs y las redes sociales, grupos de discusión periódicos sobre temas concretos, hasta implementar un consejo consultivo con los representantes de empresas y asociaciones locales y otros miembros destacados de la comunidad.

\subsection{Washington Public Library (District of Columbia, EUA)}

El Plan of Service 2013-2016 de la Washington Public Library fue creado a partir de las opiniones de los stakeholders recogidas en dos reuniones:

En la primera reunión se realizó un análisis DAFO (debilidades, amenazas, fortalezas, oportunidades), sobre la ciudad de Washington. Posteriormente se debatió sobre cuál debería ser la visión futura de la biblioteca, y a partir de las diferentes declaraciones se elaboró una lista de las necesidades.

En la segunda reunión se presentaron 18 posibles servicios que respondían a las necesidades expuestas en el encuentro anterior. En la primera fase, de los 18 servicios presentados los stakeholders escogieron 8 servicios que fueron ampliamente debatidos. Finalmente se redujeron a 3 los servicios determinando así los puntos estratégicos de los próximos años que se citan a continuación:
- Crear jóvenes lectores: alfabetización de niños;

- Estimular la creatividad y la expresión de la imaginación: leer, ver, escuchar y compartir contenidos de ocio;

- Satisfacer la curiosidad y las necesidades informativas: aprendizaje continuo.

\subsection{Bellingham Public Library (Washington State, EUA)}

El plan estratégico 2013-2017 es el resultado de las opiniones recogidas a través de:

- Entrevistas telefónicas.

- Jornadas de puertas abiertas.

- Grupos de discusión.

El día 17 de abril de 2012 se reunieron los representantes de 40 stakeholders: departamentos de la administración, ayuntamiento, otras bibliotecas, sistema de escuelas públicas, antiguos miembros del Consejo Consultivo de la Biblioteca, asociación de amigos de la biblioteca y un gran número de asociaciones y empresas locales. La reunión se inició con una declaración por parte del director de la biblioteca sobre las bibliotecas públicas y sus principales funciones en el siglo XXI y sobre la importancia de la participación de los stakeholders en la planificación estratégica. Posteriormente se dividieron los 40 stakeholders en grupos más pequeños y se procedió a un debate sobre la biblioteca, siguiendo las cuatro dimensiones (de polos confrontados) propuestas por la American Library Association (ALA):

- Biblioteca física - Biblioteca digital.

- Centrada en el individuo - Centrada en la comunidad.

- Colección - Creación.

- Portal - Depósito.

Se solicitó a los presentes que conversaran sobre estos temas y que optasen por uno de los polos opuestos, con el propósito de conocer qué orientación debería tomar la biblioteca (figura 3).

Finalmente se debatió sobre cuales deberían ser los valores y la misión futura de la biblioteca.

\subsection{Toronto Public Library (Ontario, Canadá)}

El plan estratégico 2012-2015 es fruto de "un amplio proceso de consulta pública" (2012, p. 6), que se llevó a cabo a través de un extenso conjunto de métodos de relación con

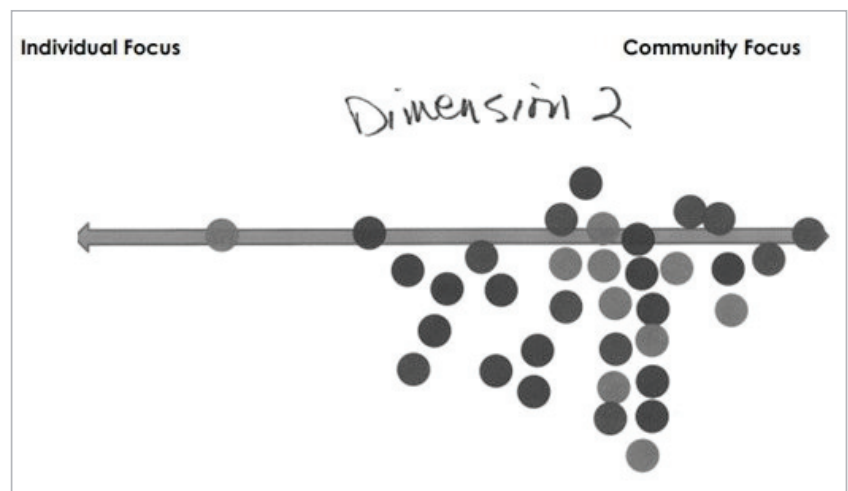

Figura 3. Graduación de la opinión de los stakeholders en la dimensión "Centrada en el individuo - Centrada en la comunidad". Fuente: Bellingham Public Library, 2012. 
las diferentes categorías de stakeholders:

- Análisis del sitio web.

- Priorización de las áreas y objetivos más significativos para la biblioteca con miembros del ayuntamiento.

- Grupos de discusión con los representantes de otras administraciones y ONGs locales.

- Consultas de los eventos de la biblioteca y la ciudad.

- Grupos de discusión con trabajadores y representantes sindicales.

- Análisis de los comentarios en los blogs y redes sociales y de los chats en Twitter.

- Grupos de discusión enfocados a la biblioteca con personas mayores y asociaciones locales, sobre el tema "Ios niños y la lectura".

La información recogida fue utilizada para crear el plan estratégico, en base a las siguientes cuatro cuestiones:

1. ¿Qué nivel de servicio necesita la biblioteca para poder atender a las necesidades de la comunidad y de los residentes, tanto ahora como en el futuro?

2. ¿Cuáles son las prioridades de los residentes para el servicio bibliotecario?

3. ¿Las prioridades del proyecto abordan todo el rango de las necesidades de los residentes?

4. ¿Cómo puede la biblioteca asegurar que los servicios sean innovadores, eficientes y sostenibles?

\section{Conclusiones}

En los últimos años, probablemente debido al contexto socio-económico, ha habido una creciente aplicación de la "teoría de los stakeholders" en otras áreas ajenas a las del sector comercial. Este hecho normalmente ha estado asociado a políticas de responsabilidad social y sostenibilidad de las instituciones, y al progresivo aumento de proyectos y movimientos de promoción de una ciudadanía participativa.

En el contexto de la península Ibérica no se puede aún hablar de un movimiento ciudadano cohesionado y efectivo, ni de instituciones totalmente abiertas a la ciudadanía, pero ya se están dando los primeros pasos.

Las bibliotecas públicas deben tener en cuenta la información que deriva del contacto directo con los grupos de interés, pues "no contar con estos datos produce un vacío entre la oferta del servicio de la biblioteca y lo que el usuario realmente necesita" (González-Fernández-Villavicencio, 2012).

Es necesario que estos movimientos se basen en la investigación científica, y adopten y apliquen las técnicas y principios que permitan establecer relaciones entre los stakeholders y la institución. De otro modo los proyectos y movimientos de participación ciudadana y responsabilidad social corporativa se convierten en una lista de buenas intenciones y algunos proyectos sueltos sin resultados reales a largo plazo, es decir, sin un verdadero cambio en la gestión de las organizaciones.

\section{Bibliografía}

AccountAbility (2006). El compromiso con los stakeholders: Manual para la práctica de las relaciones con los grupos de interés. Stakeholder Research Associates / Instituto Nóos. http://www.accountability.org/images/content/2/0/204.pdf http://www.accountability.org.uk

Belio-Galindo, José-Luis; Sainz-Andrés, Ana (2007). Conozca el nuevo marketing: el valor de la información. Madrid: Especial directivos. ISBN: 9748493590215

Bellingham Public Library (2012). Stakeholder retreat, April 17.

http://www.bellinghampubliclibrary.org/News/images/ StakeholdersRetreatSummary.pdf

Bellingham Public Library (2013). Connecting our community with each other and the world: read, learn, meet, discover: Strategic plan 2013-2017.

http://www.bellinghampubliclibrary.org/about/ strategicplan2013-2017.pdf

Conamaj. ¿Qué es participación ciudadana?

http://www.conamaj.go.cr/index.php/proyectos/ participacion/14-ppc/48-definicion

Freeman, Edward (1984). Strategic management: a stakeholder approach. Boston: Pitman. ISBN: 0273019139

González-Fernández-Villavicencio, Nieves (2012). "Servicios de referencia en bibliotecas universitarias: tendencias y plan de marketing". El profesional de la información, v. 21, n. 6, noviembre-diciembre, pp. 567-576.

http://dx.doi.org/10.3145/epi.2012.nov.03

Granda-Revilla, Germán; Trujillo-Fernández, Ricardo (2011). "La gestión de los grupos de interés (stakeholders) en la estrategia de las organizaciones". Economía industrial, n. 381, pp. 71-76.

http://www.minetur.gob.es/Publicaciones/Publicacionesperiodicas/ Economialndustrial/RevistaEconomialndustrial/381/ Germ\%C3\%A1n\%20Granda\%20Revilla.pdf

Toronto Public Library (2012). Read, learn, create, deliver: Strategic plan 2012-2015.

http://www.torontopubliclibrary.ca/content/about-thelibrary/strategic-plan/pdfs/read-learn-create-deliver-stratplan-2012-2015.pdf

Washington Public Library (2013). Plan of services 20132016.

http://www.washington.lib.ia.us/library-information/Irp2

Wicks, Andrew; Gilbert, Daniel; Freeman, Edward (1994). "A feminist reinterpretation of the stakeholders concept". Business ethics quarterly, v. 4, n. 4, pp. 475-497.

http://dx.doi.org/10.2307/3857345 